\title{
Amphiphilic Molecules and Liquid Crystals
}

\section{Citation}

Pershan, Peter S. 1979. Amphiphilic molecules and liquid crystals. Journal de Physique 40(C3): 423-432.

\section{Published Version}

doi:10.1051/jphyscol:1979385

\section{Permanent link}

http://nrs.harvard.edu/urn-3:HUL.InstRepos:10361923

\section{Terms of Use}

This article was downloaded from Harvard University's DASH repository, and is made available under the terms and conditions applicable to Other Posted Material, as set forth at http:// nrs.harvard.edu/urn-3:HUL.InstRepos:dash.current.terms-of-use\#LAA

\section{Share Your Story}

The Harvard community has made this article openly available.

Please share how this access benefits you. Submit a story.

Accessibility 


\title{
AMPHIPHILIC SYSTEMS.
}

\section{AMPHIPHILIC MOLECULES AND LIQUID CRYSTALS}

\author{
P. S. PERSHAN \\ Division of Applied Sciences, Harvard University, Cambridge, Mass. 02138, U.S.A.
}

\begin{abstract}
A brief description of the essential features of amphiphillic molecules will be presented. This will be followed by a discussion of specific physical phenomena and their relation to a number of contemporary research areas. Foremost amongst these is the possible application of amphiphillic monolayers to the physics of two-dimensional systems. Specific experimental and theoretical examples will largely be drawn from studies of synthetic phosphatidylcholine (e.g., lecithin) type lipids. Recent experimental results from our laboratory will be mentioned.
\end{abstract}

1. Introduction. - The intention in preparing this article is to provide a brief survey of some of the salient features of amphiphillic materials and then illustrate how the physical properties of macroscopic systems formed from amphiphillics relate to phenomena of fundamental importance to a variety of other subjects. Hopefully, this superficial discussion of diverse phenomena from disparate disciplines will enable the reader that is already an expert in one discipline to better appreciate how his work bears on the current activities in other disciplines. The reader should also be aware that this paper was originally prepared for oral presentation and, in the manner of oral presentations, there are fewer literature citations than would have been the case had this article been prepared as a written review article. Nevertheless those citations that are listed should provide the reader with entry points into the literature of different fields. One of the classical textbooks on amphiphillic materials is Adamsons' [1], however, Tanford has recently prepared an excellent and concise review [2] of hydrophobic effects and his own book [3] is certainly relevant.

2. Amphiphillic molecules. - The saturated fatty acid $\mathrm{CH}_{3}\left(\mathrm{CH}_{2}\right)_{n} \mathrm{COOH}$ can be considered as the prototype of all amphiphillic molecules in that it consists of two parts which separately have very different solubilities in water, or other solvents. In this case the polar part, $\mathrm{HCOOH}$ or formic acid, is infinitely soluble in water while the alkane or hydrocarbon part $\mathrm{CH}_{3}\left(\mathrm{CH}_{2}\right)_{n-1} \mathrm{CH}_{3}$ is much less soluble in water than in most non-aqueous solvents. For simple alkanes, with less than $10 \sim 20$ carbon atoms, the solubility in water decreases by roughly one order of magnitude with each increase in the carbon number by two. Conventional terminology attributes this decrease with increasing size of the hydrocarbon to a hydrophobic interaction. Unfortunately this is somewhat of a misnomer since the effect does not derive from a repulsion between water and the hydrocarbon $[2,3]$. Rather it is well known that when a hydrocarbon is in contact with water the network of hydrogen bonds between water molecules reconstructs itself to avoid the region occupied by the hydrocarbon. This constraint on the local structure of water decreases the entropy of the water near the hydrocarbon and results in a larger free energy for the total system, i.e., the water plus the solvated hydrocarbon. The hydrophobic effect thus arises more from the self-attraction of water for itself, which tends to squeeze the hydrocarbon out, rather than a repulsion between water and hydrocarbon. A corollary of this is that for hydrophobic groups more complicated than the simple saturated normal alkanes mentioned above, i.e., those containing branched chains or those with large enough carbon numbers that the chain can fold over upon itself, the solubility decreases in proportion to the surface area rather than the carbon number.

Amphiphillic molecules are often termed surfactants or surface active molecules. Figure 1 illustrates that if one dissolves a low concentration of amphiphillics in a water solution, by virtue of the hydro- 


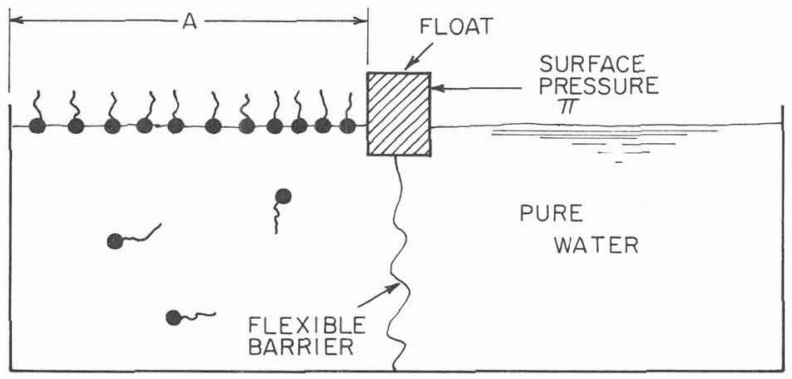

FIG. 1. - Schematic illustration of monolayer surface pressure. The float isolates the part of the surface containing surfactant from the rest. The flexible barrier isolates the volume containing surfactant from the rest of the liquid. The barrier is constructed such that the float can move without changing the relative volumes on either side. Also it does not exert a lateral force on the float.

phobic effect a certain fraction of the molecules will partition between the surface and the bulk with their tails pointed away from the water. Gibbs discussed this effect over a century ago [4]. The watersurfactant surface has lower energy or surface tension than for pure water. Thus in the experimental geometry schematically shown the float on the surface will move to the right in order to lower the total surface energy of the combined system. The flexible barrier allows this to occur without changing the depth on either side. The float can be kept stationary by application of a force proportional to the length of the line of contact between the float and the surface. Thus one speaks of a surface pressure $\pi$ with the dimensions of force per unit length that might be interpreted as the two dimensional analogue of the normal three dimensional pressure. Gibbs showed that in equilibrium with the solution $\pi$ is a function of the ambient three-dimensional pressure, the temperature and the chemical potential of the amphiphillic molecules in solution, i.e., $\pi(T, P, \mu)$, with the inequality $(\partial \pi / \partial \mu)_{T, P} \geqslant 0$. Since one also can write $\mu=\mu_{0}+k_{\mathrm{B}} T \ln X$ where $X$ is the activity of the amphiphillic in the bulk (for low concentrations $X$ is proportional to the concentration) the surface pressure should be a monotonically increasing function of concentration. The unitary potential of the amphiphillic, $\mu_{0}$, reflects the amphiphillic character of the molecule. For larger hydrocarbons $\mu_{0}$ is larger and more positive, for polar parts with stronger tendencies to dissolve in water $\mu_{0}$ is more negative. The relative strengths of these two effects determines what is known as the HLB or hydrophillic-lipophillic balance for a given molecule.

Experimentally $\pi$ does increase monotonically with increasing concentration only so long as $X$ is less than some number known as the critical micelle concentration or CMC [i.e., $X=X(\mathrm{CMC})]$. For this concentration $\mu=\mu_{0}+k_{\mathrm{B}} T \ln X(\mathrm{CMC})$ the chemical potential of the monomer in solution becomes equal to the chemical potential for some phase in which the molecules aggregate. The aggregated phase acts as a sort of buffer and $\mu$ becomes fixed implying that $\pi$ is also fixed. In the case of the fatty acid, and also for many other amphiphillics this aggregated phase consists of a solution of micelles as illustrated in figure 2.

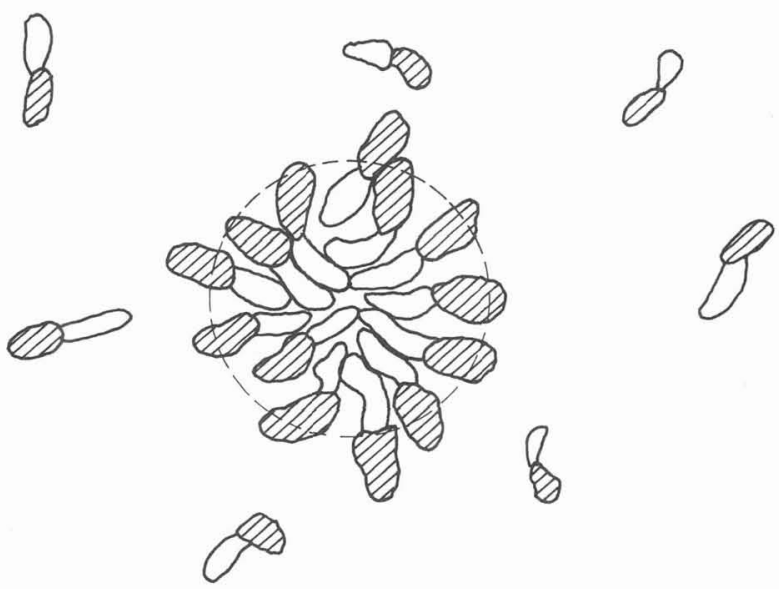

FIG. 2. - A cross-section view of the molecular packing at some instant in time for amphiphillic molecules in a micelle containing approximately 50 monomers. The cross-hatched areas indicate the polar or hydrophillic part of the molecule. Micelles are in dynamic equilibrium with the monomer solution and the structure is not static.

Typical micelles are aggregates of from 20100 monomers in dynamic equilibrium with the monomer solution. They organize themselves such that the polar part forms a sort of barrier that separates the hydrocarbon part from the water. The hydrophobic effect, is essentially shielded so that although the chemical potential for the molecules in the micelle becomes a complicated function of intermolecular, intramolecular, and solvent interactions the physical state of the water surrounding the micelle is not directly affected by the hydrocarbon. Very interesting questions arise as to the precise size and shape of the micelle and the physical phenomena that determine them. We will not discuss these here except to point out that in order to pack the hydrocarbon part of these amphiphillics into the micelle structures the chains must be somewhat flexible. Alternatively if the hydrocarbon parts are too bulky when compared with the size of the polar part micellar packing will not be possible.

The first of these effects is well illustrated by considering the phase diagrams of simple soaps. Skoulius has written a concise review of this field and figure 3 summarizes some of the general properties of soaps [5]. The simplest soaps are just alkali salts of saturated fatty acids, i.e., a potassium soap of the form $\mathrm{CH}_{3}\left(\mathrm{CH}_{2}\right)_{n} \mathrm{COOK}$. Above some temperature $T_{\mathrm{c}}$, at high water content the soaps exist as micellar solutions. For lower water content there is not sufficient water to fill the space between spherical, or near spherical micelles, and the aggregated phases 

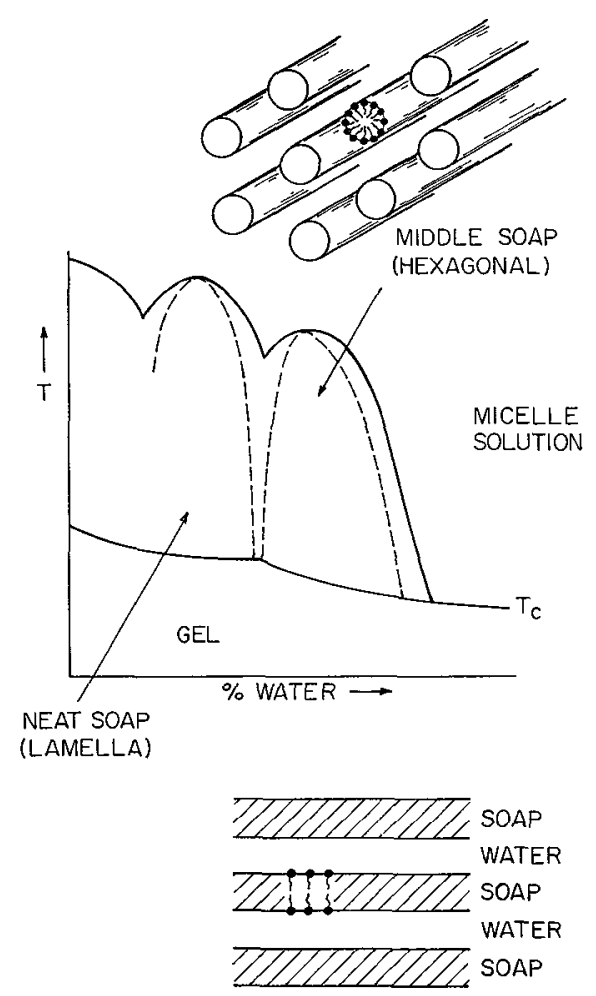

FIG. 3. - Illustration of a typical phase diagram for a simple soap. See Skoulius [5] for further details.

are either cylindrical (middle phase) or lamella (neat phase). Skoulius discusses variations on these simple forms but we will not go into those details here. The interesting feature of this phase diagram is that in the middle phase, in the neat phase, and in general for $T>T_{\mathrm{c}}$ a variety of experiments demonstrate that the hydrocarbon parts of the molecules are relatively flexible or fluid like. In contrast for $T<T_{\mathrm{c}}$ the hydrocarbon parts are relatively rigid or solid like. Consequently one expects, and experiments demonstrate that for $T<T_{\mathrm{c}}$ micelles will not form because the rigid molecules will not easily pack into the micelle structure. Since micelles in solution tend to scatter light relatively strongly micellar solutions have a somewhat milky appearance. On lowering the temperature below $T_{\mathrm{c}}$ there is a sudden clearing of the solution as the micelles become unstable and form larger aggregates that percipitate out of the solution. The temperature at which this occurs is often referred to as the Kraft point.

In addition to the simple fatty acids and their alkali salts (the soaps) there are a much larger variety of amphiphillic molecules than we want to review here. For example, a common amphiphillic used in shampoos has a sulfate for the polar part

$$
\mathrm{CH}_{3}-\left(\mathrm{CH}_{2}\right)_{n}-\mathrm{O}-\left(\mathrm{SO}_{3}^{-}\right) \mathrm{K}^{+}
$$

Aerosols, such as the common foaming shaving creams, often contain powerful wetting agents such as the dialkyl ester of sulfosuccinic acid<smiles>CCCCCC(=O)OCCC</smiles>

in which there are more than one hydrocarbon chain. Derivatives of ethylene oxide

$$
\mathrm{CH}_{3}-\left(\mathrm{CH}_{2}\right)_{n}-\left(\mathrm{O}-\mathrm{CH}_{2}-\mathrm{CH}_{2}\right)_{m} \mathrm{OH}
$$

are of yet a different type. The list could obviously be extended, however in the rest of this article we will concern ourselves with one special amphiphillic of biological significance, namely the di-acylphosphoglyceride or phospholipid<smiles></smiles>

where $\mathrm{R}_{1}$, and $\mathrm{R}_{2}$ refer to hydrocarbon chains and $X$ refers to one or another smaller chemical group. If $\mathrm{R}_{1}$ and $\mathrm{R}_{2}$ are both of the form $\mathrm{CH}_{3}\left(\mathrm{CH}_{2}\right)_{14}^{-}$this is referred to by the prefex di-palmitoyl. If $X$ is of the form $-\mathrm{CH}_{2}-\mathrm{CH}_{2}-\mathrm{N}\left(\mathrm{CH}_{3}\right)_{3}^{+}$the molecule is called dipalmitoyl phosphatidyl choline or DPPC. Shorter hydrocarbon chain lengths, obtain other lipids. For example if both chains have the form $\mathrm{CH}_{3}\left(\mathrm{CH}_{2}\right)_{12}$ one has dimyrystoyl-phosphatidyl choline or DMPC.

Variations with different polar parts, different chain lengths, two unequal chains, chains with double bonds etc. are all possible and all are referred to as phospholipids. Although all amphiphillics exhibit interesting physical properties the lipids are of particular interest to us here because : 1) they play a major role in the architecture of biological membranes, 2) their amphiphillic character is essential to this role, and 3) macroscopically oriented single domain lamella or smectic liquid crystals are readily formed from these molecules.

3. Lipids and biological membranes. - The accepted picture of biological membranes is the fluid mosaic model illustrated schematically in figure 4 [6]. The lipid molecules organize themselves into a bilayer structure in which the hydrophillic polar parts shield the hydrophobic hydrocarbon part from the aqueous environment surrounding the membrane. A large variety of important biological ingredients are relatively insoluble in hydrocarbons and the bilayer forms an impenetrable barrier to those ingredients. Proteins are attached to the bilayer, either spanning the entire thickness or protruding from only one side and it is the action of these proteins that convert an otherwise inactive structure into a living membrane. 


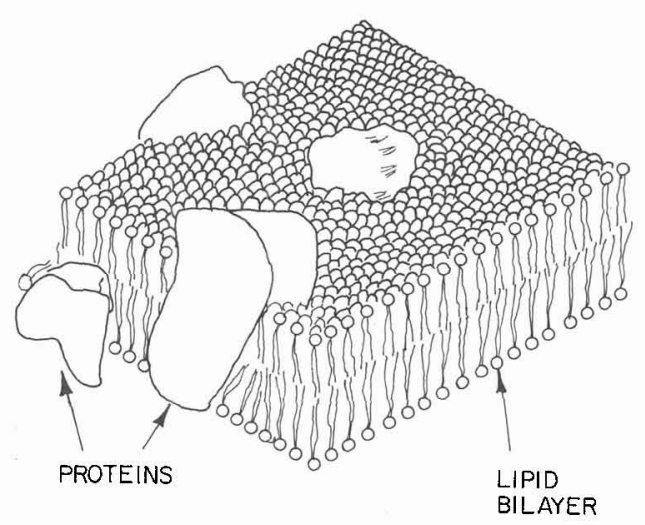

FIG. 4. - A schematic view of the fluid mosaic model of biological membranes [6]. Proteins sit in and on the lipid bilayer as shown. In addition there are extensive extra membraneous structures that are not shown but which are essential to membrane function.

For example the protein controlled flux of protons though mitochondrial membranes is one key ingredient in the conversion of ADP to ATP.

Proteins make membranes work but the proteins exist in and on an amphiphillic lipid bilayer and depend on the physical properties of that bilayer to function. Hinkle and McCarty's article How Cells Make ATP in Scientific American gives a very readable picture of some of the complex chemical and physical processes taking place in and on membranes [7].

The recent physical studies on lipids have been briefly reviewed by Nagle and Scott in Physics Today [8]. A slightly older review, from a somewhat different point of view is the article by Dennis Chapman [9]. Figure 5 illustrates essential features of the phase diagram for DPPC-water mixtures. There are both similarities and differences to the phase diagram for simple soaps shown in figure 3. First and foremost amongst the similarities is the existence of a first order phase transition separating regions in which the hydrocarbons are rigid or solid-like from the regions where they are flexible or fluid-like. In figure 3 this was indicated by the $T_{\mathrm{c}}$ line. In the case of lipids the transition is referred to as the gel transition and the temperature at which it occurs is indicated by $T_{\mathrm{g}}$. For both lipids and soaps, as well as for all other amphiphillics the temperature of the gel transition decreases with increasing water content. A significant difference between lipids and soaps is the high water region. At temperatures above $T_{\mathrm{c}}$, or above the gel transition, and at high water content the soaps form micellar solutions. Micelles do not form for DPPC although they are observed for similar lipids with hydrocarbon chain lengths containing fewer than ten $\left(\mathrm{CH}_{2}\right)$ groups. Presumably two hydrocarbon chains, each containing fourteen $\mathrm{CH}_{2}$ groups are too bulky for micellar packing. Thus if $T>T_{\mathrm{g}}$, for weight fractions greater than $40 \%$ (water to total sample weight) DPPC water mixtures separate into one

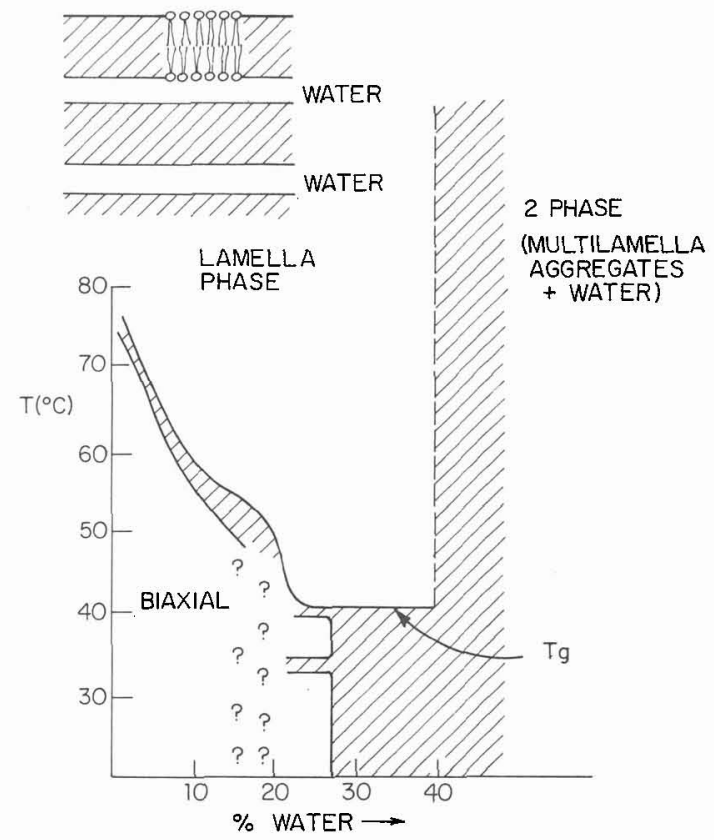

FIG. 5. - Simplified version of the phase diagram for DPPC, i.e., dipalmitoyl phosphatidylcholine-water mixtures. On cooling the onset of the gel transition is indicated by the line $T_{\mathrm{g}}$. Many details of the phase diagram below $T_{\mathrm{g}}$, although known, are omitted for simplicity. The calibration of the horizontal axis is percentage of water to total sample weight.

phase that is essentially pure water (i.e., less than $\sim 10^{-10}$ molar in DPPC) and a lamella or smectic $A$ phase as shown in figure 5 .

It is interesting to note that for the soap (Fig. 3) the lamella phase is separated from the micellar phase by a hexagonal phase. The hexagonal phase does not form under similar conditions for DPPC, presumably for the same reason that DPPC does not form micelles, because the relative size and shape of the hydrocarbon to polar part make the packing unfavorable. We will return later to discuss the role of water in stabilizing the lamellar phase of DPPC and the reasons for the phase boundary at $40 \%$ weight water.

Since real biological membranes contain a number of different lipids, each of which have gel transitions at different temperatures when pure, it is not surprising that in biological membranes the gel transition is spread out over $20 \sim 30^{\circ} \mathrm{C}$. Melchior and Steim [10] have reviewed the evidence for thermotropic phase transitions in biological membranes. Some of the most interesting experiments result from a bacterium $A$. laidlawii that can be obtained in a mutated form which is incapable of synthesizing the fatty acids needed to form the lipids that are necessary for its own membranes. Thus, if these bacteria are grown under conditions in which one supplies them with short chain fatty acids the membranes of the bacterial will contain only lipids with short hydrocarbon chains and low gel transition temperatures. If the bacterium are fed longer chain fatty acids the mem- 
branes will have higher gel transition temperatures. In this way bacteria can be obtained in which gel transition temperatures vary by as much as $70^{\circ} \mathrm{C}$.

Studies such as the temperature dependence of enzyme activity, respiration rate, growth rate, etc. all show marked changes that correlate with the gel transition. Without overstating the case it is safe to say that there is evidence that the physical state of the lipids play a role in biological processes. Although the practical importance of this for regulatory life processes, or for realization of diverse properties within a single membrane, is not yet agreed upon one can justify research into the physical properties of lipids with the assurance that these properties do play some role. The remainder of this article will deal with physical properties of lipid systems.

4. Physical studies of lipid systems. - One of the earliest techniques employed in the study of lipid systems is based on the tendency of amphiphillics to form surface monolayers of the type illustrated schematically in figure 1 [11]. An important difference between lipids such as DPPC with very strong hydrophobic parts and the soaps discussed earlier is the fact that DPPC does not form micelles and the solubility for the monomer is less than $10^{-10}$ molar under usual circumstances. Thus if a monolayer of DPPC is formed at a water air interface there is hope that the system can be regarded as being truly two-dimensional with a fixed number of molecules. According to Gibbs [4], and numerous experimental studies [9], at low densities (molecules $/ \mathrm{cm}^{2}$ ) the surface acts as a nearly perfect two-dimensional gas, e.g., $\pi A \propto N T$. At higher density, i.e., larger values of $N / A$, there is also evidence for a phase transition in which the two-dimensional gas condenses into a two-dimensional liquid. The interesting question is whether one can also realize two-dimensional crystalline or liquid crystalline phases by suitable choice of molecular species, surface pressure $\pi$, density $N / A$, or temperature. Dervichian's early work [11], as well as more recent studies all suggest this is possible and a recent publication [12] presents the case supporting this suggestion in great detail. Figure 6 is a schematic version of the phase diagram proposed for monolayer films of DPPC at a water air interface.

According to Alrecht et al. the $\pi$ - $T$ plane for DPPC is separated into four regions or phases; a twodimensional isotropic fluid, an anisotropic twodimensional fluid or two-dimensional liquid crystal, and two separate crystalline regions. As for threedimensional isotropic fluids the region of the twodimensional isotropic fluid contains a line marking the liquid-gas coexistence condition. This line terminates at a critical point CP1. They also indicate a second critical point CP2 along the coexistence line separating the anisotropic fluid from one of the crystalline regions. Presumably at higher surface pressures $\pi$ the transition from crystal II to aniso-

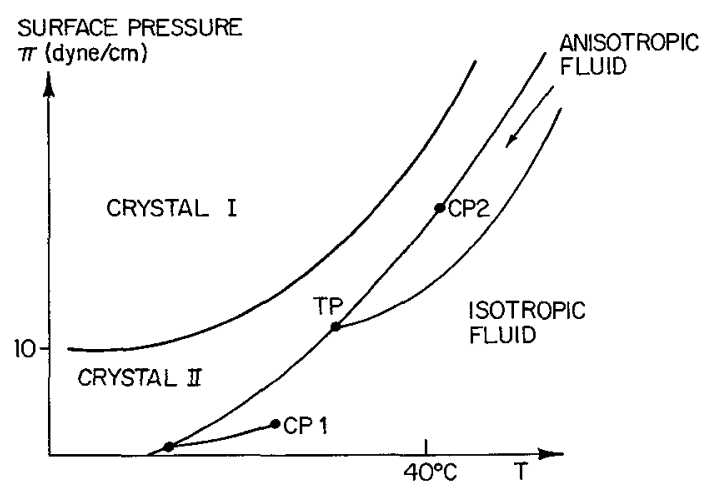

FIG. 6. - Schematic version of the two-dimensional phase diagram for insoluble monolayers of DPPC at a water air interface. This form was proposed by Albrecht et al. [12]. The surface pressure of 10 dynes/cm and temperature of $40^{\circ} \mathrm{C}$ occur where indicated.

tropic fluid is second order while for lower pressures it is first order. A triple point TP marks the coexistence point of the crystal, the isotropic fluid and the anisotropic fluid. If they are right, such that this variety of two-dimensional phases can be observed with amphiphillic monolayers in general, and for lipids in particular, amphiphillics will certainly play an important role in the current research activities on phase transitions in two dimensions. Below we will return to discuss some of the difficulties encountered when studying monolayers at high surface pressures and explain why we raise the question "If they are right! » in relation to figure 6 . Before that, however, we will discuss some aspects of phase transitions in two dimensions.

Although the concept of a two-dimensional crystal appears straight-forward there are basic theoretical results which introduce subtle complications. For example one speaks of long range order in a threedimensional crystal by defining a set of three unit vectors $\left\{\bar{a}_{i}\right\} ; i=1,2,3$. It then follows that aside from small fluctuations the vector connecting any two lattice points can be expressed as a linear combination of the $\left\{\bar{a}_{i}\right\}$ with integral coefficients, e.g., $\bar{R}_{n}=n_{1} \bar{a}_{1}+n_{2} \bar{a}_{2}+n_{3} \bar{a}_{3} ;\left\{n_{i}\right\}=$ integers. It is straightforward to show that this is not true for a two-dimensional crystal, since the thermodynamic fluctuations in the relative positions of two lattice points grow in proportion to the logarithm of the distance between the two points $[13,14]$. Thus for an infinitely large two-dimensional crystal one cannot speak of the same type of long range order, or periodicity, that one has for a three-dimensional crystal. Nevertheless there is general agreement that suitable definitions of the order allow one to speak of two-dimensional crystals. Similar issues arise in the discussion of long range order in three-dimensional smectic $A$ phases $[15,16]$. The important point is that subtle differences exist between the theory of phases and phase transformations in the two and three dimensions and these differences have stimulated considerable research activity. 
Studies of monoatomic or monomolecular layers deposited on crystalline substrates constitute what is probably the principle experimental effort into twodimensional crystalline order. Berker et al.'s theoretical paper on this subject cites most of these recent experimental articles and also describes some of the phase diagrams [17]. In lieu of going into that literature in any sort of detail we will restrict ourselves to a few remarks on the recent theory by Halperin and Nelson on the nature of melting in two dimensions [18]. According to them the two-dimensional crystal at finite temperature will always have a finite concentration of lattice vacancies that can be thought of as pairs of opposite dislocations. In the crystalline state these dislocations are bound to each other with a finite energy. Halperin and Nelson demonstrate that at some finite temperature these dislocations become unbounded of each other and move freely throughout the two-dimensional crystal.

They interpret this as the melting of the twodimensional crystal. Since it is well known that free mobile dislocations render a system incapable of sustaining static shear stresses the elastic properties of the melted phase will be very much like that of a two-dimensional fluid. The transition might profitably be thought of as the elastic analogue of the transition from a normal to a superfluid [19]. In any event the phase that occurs on melting is not a simple two-dimensional isotropic fluid for which the orientational correlation functions between bond directions decay as an exponential function of distance. Rather this phase is analogous to a two-dimensional nematic liquid crystal in which bond directions play the same role that molecular axes play in conventional nematics. In contrast to three dimensional nematics, director fluctuations, e.g., $\int\left(k_{\mathbf{B}} T / K q^{2}\right) \mathrm{d}^{2} q$, are not integrable and there is no true long range orientational order in two dimensions. In this phase the orientational correlation functions decay algebraically with distance and there will be a second phase transition at a higher temperature than the crystal melt in which the correlations switch from algebraic decay to the exponential decay expected for a simple two-dimensional fluid. In view of the similarity between this progression of phases and the phase diagram proposed by Albrecht et al. [12] for DPPC monolayers (Fig. 6) it is very important that one critically evaluate both the DPPC experimental results and the appropriateness of the Halperin-Nelson model for DPPC.

To consider the experimental results first, Gershfeld and Tajima [20] pointed out a basic difficulty with all monolayer studies. In their experiment, illustrated schematically in figure $7 a$, a three-dimensional sample of DMPC (either solid or liquid crystalline) is put in contact with a clean air-water interface. DMPC differs from DPPC by having two less $\mathrm{CH}_{2}$ groups in each of the hydrocarbon chains.

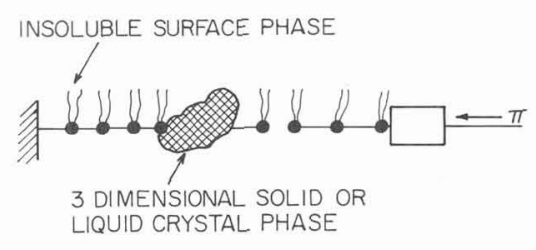

a)

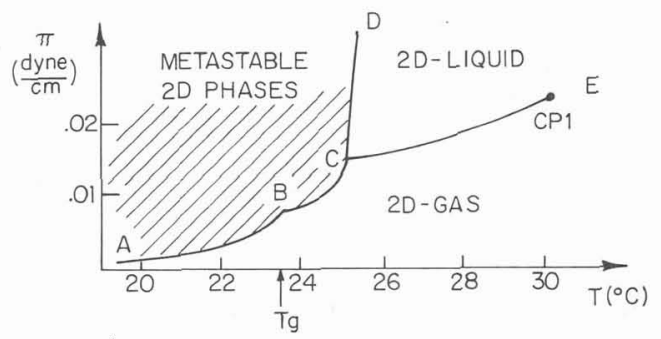

b)

FIG. 7. - a) Schematic illustration of the Gershfeld-Tajima [20] experiment. The amphiphillic forms an insoluble monolayer in equilibrium with the 3-dimensional phase. b) The phase diagram obtained by Gershfeld and Tajima for DMPC. In the text we discuss the disparity in the surface pressures shown here and in figure 6 .

Although the gel transition for DMPC is lower than for DPPC the two molecules have essentially the same phase diagram. In a process reminescent of evaporation, molecules from the three-dimensional phase spread out on the surface until the chemical potential for the surface molecules become equal to the chemical potential of the molecules in the threedimensional phase. As we mentioned earlier, in thermal equilibrium at a given $P$ and $T$, the surface pressure $\pi$ is uniquely given by the chemical potential $\mu$. The line $A B$ in figure $7 b$ is the $\pi$-T line when the surface film is in equilibrium with the threedimensional phase in which the hydrocarbons are stiff or solid like. For $T>T_{\mathrm{g}}$ the film is in equilibrium with a three-dimensional phase in which the chains are fluid like. If the three-dimensional phase is removed one can expand the area of the film and measure the $\pi-A$ isotherms at constant number of surface molecules. For the expanded film these would all be to the right of the ABCD line. The line CE represents the two-dimensional liquid-gas coexistence line. It terminates in the critical point E. If, after removal of the three-dimensional phase, one attempts to compress the film rather than expanding it one enters the cross-hatched region to the left of $\mathrm{ABCD}$. Elementary thermodynamics is sufficient to demonstrate that in this region the chemical potential $\mu(P, T, A)$ is larger than the chemical potential along the line $\mathrm{ABCD}$ for the same $P, T$. Thus at any point in the cross-hatched region the chemical potential of the monolayer is higher than the chemical potential for one of the three-dimensional phases that could exist at the same $T$ and $P$. Under 
this situation the monolayer is metastable and great care must be exercised when interpreting $\pi-A$ measurements to insure that an apparent phase transition is really a transition between two different twodimensional phases and not a transition in which a metastable two-dimensional phase " condenses » into a mixture of some three-dimensional phase and a monolayer phase of lower free energy. This is particularly serious for DPPC since in some ranges of temperature the surface pressures observed by Gershfeld and Tajima to separate the metastable and stable regions of the phase diagram are two to three orders of magnitude lower than the surface pressures at which phase transitions are suggested in figure 6 . To be absolutely clear, we are not arguing the phase diagram obtained by Albrecht et al. [12] is wrong. Rather we are suggesting that all experimental studies of, what one hopes are, two-dimensional systems must be carefully evaluated to determine that they truly are two-dimensional.

Returning to the theoretical studies appropriate to DPPC, although the principle features of the Halperin, Nelson work are probably relevant these authors were not trying to develop a model for amphiphillic monolayer systems. Thus the monomer units in their two-dimensional model had neither internal structure nor other degrees of freedom aside from the positions of their centers of mass. On the other hand, we know that the flexibility of the chains and the chain melting transitions are important to threedimensional phase diagrams and they must also be important for some features of the two-dimensional phase diagram. The article by Nagle and Scott in Physics Today reviews some of the recent results [8].

For example, Nagel developed a mathematical model that relates the flexibility of the hydrocarbon to both the temperature and mean surface area per hydrocarbon chain. For this model he calculates the surface pressure $\pi$ that corresponds to this temperature and surface area and predicts the monolayer $\pi-A$ isotherms. He also predicts a first order transition from a phase of flexible to straight chains at low $\pi$ and a second order transition at higher $\pi$. The critical point in the phase diagram proposed by Albrecht et al. (see Fig. 6) along the boundary between the crystalline and anisotropic fluid phases corresponds to this. It is interesting to note that the temperature of this critical point appears to be very close to the temperature $T_{\mathrm{g}}$ of the gel transition observed in the three-dimensional smectic phases (see Fig. 5) at high water. This lead Nagel to argue that the molecular details in the bilayers making up the three-dimensional phases are essentially the same as the molecular details of the molecules in the monolayer at the temperature and surface pressure corresponding to the critical point.

If the concern expressed by Gershfeld and Tajima about the metastability of monolayers at high surface pressure does not prove to be a fundamental obstacle it would be very interesting to study the effect of chain length on the high surface density part of the two-dimensional phase diagram.

For example, as discussed in the Nagel-Scott review article, the first few carbon-carbon bonds, nearest to the polar part of the amphiphillic, are constrained by the position and orientation of the polar part at the interface. By virtue of cis-trans isomerization and other thermal effects the orientation of bonds further down the chain become more and more random. Marcelja [21], Bothorel et al. [22] and others have developed theoretical models that reproduce some of the experimental results for this effect [23, 24]. All agree that $\left(\mathrm{CH}_{2}\right)$ groups more than approximately $10 \mathrm{C}-\mathrm{C}$ bonds away from the polar part are essentially randomly oriented. This must surely have an effect on the short range repulsive forces between surface molecules. Furthermore, for DPPC and many other amphiphillics as well, the polar part of the molecule is not cylindrically symmetric. Coupling between polar group and the hydrocarbon chains can thus cause anisotropy in the chain configuration that should be sensitive to the size of the chain. All this is rather speculative but, if surface pressure can induce two-dimensional phase transitions without complicating three-dimensional effects, amphiphillic monolayers should be very interesting.

The principle contribution to come from our own laboratory has been the development of improved techniques for obtaining macroscopic, single domain, aligned, multilamella, smectic-A samples. The initial experiment to obtain aligned samples of DPPC monohydrate was done by Powers and Clark [25]. However, more recently we were able to control the amount of water and also to incorporate other ingredients into the aligned lipid bilayers [26]. These included cholesterol, various antibiotics, chlorophyll-a [26] and $\beta$-carotene [27]. This is particularly important since the individual lipid bilayer is widely used as a model biological membrane. If one can also demonstrate that the individual bilayers making up the multilamellar smectic are also good models than the ability to incorporate typical membrane ingredients into the smectic will facilitate spectrographic studies that might be impossible on these ingredients when included in either unoriented multilamellae or single bilayers.

We will not discuss the techniques for obtaining single domain samples since they can be read elsewhere $[25,26]$. We would, however, like to stress that in most of their essential properties these samples are very much like the thermotropic smectics that have been studied in many laboratories for many years. For example, Rosenblatt et al. demonstrated that above a critical strain the thermotropic smectic CBOOA deforms through the appearance of a regular array of parabolic focal conic defects [28]. We have been able to produce exactly this same array in at least three different lipids under similar condi- 
tions [29]. More recently we have been able to induce light scattering effects with much lower strains and observe phenomena that is essentially identical to the undulation instability effects well known in thermotropic smectics at small strains [30].

Although it is not easy to demonstrate that the multilamellar samples constitute acceptable models for biological membranes one can argue indirectly from available data that for more than approximately $20-25 \%$ weight water to total sample weight and for temperatures above $T_{\mathrm{g}}$ (see Fig. 5), the bilayers are not significantly perturbed by the multilamella structure. Thus if the isolated bilayer is an acceptable model, which it is, than the multilamellar should also be acceptable.

Consider the chemical potential for water in the planar spaces separating adjacent bilayers. We know that at low water content lipids are strongly hygroscopic. Thus for low water content the chemical potential for the water contained in the lipid must also be very low. Furthermore we also know-that as more water is added to the lipid it becomes less and less hygroscopic and eventually reaches an equilibrium state, which for DPPC is $40 \%$ water (see Fig. 5), when the chemical potential for water in the lipid is equal to that of bulk water. This is shown schematically in figure 8 . Obviously, if the

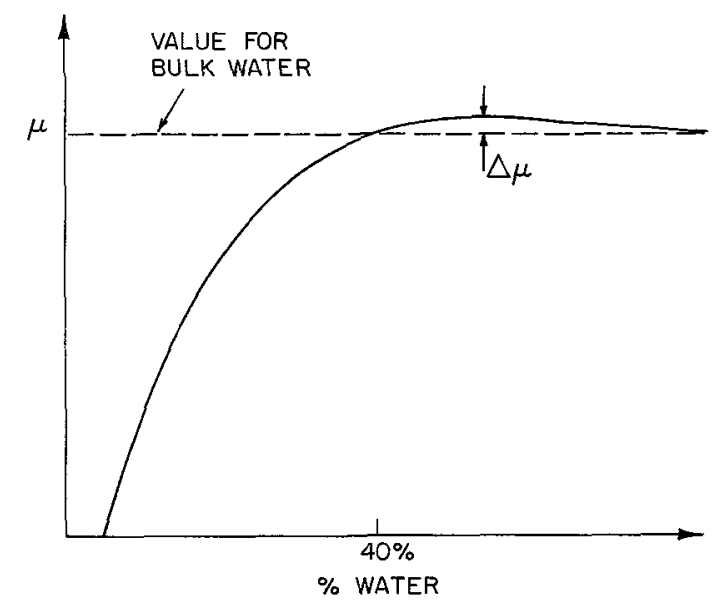

FIG. 8. - Qualitative representation of $\mu$, chemical potential for the water within the lamella phase of DPPC, as a function of water concentration. The two phase limit, shown at $40 \%$ in figure 5 occurs when the chemical potential becomes equal to that of bulk water. The quantity $\Delta \mu$ is one measure of the attractive forces between adjacent lipid bilayers in the lamella phase.

number of water molecules per lipid were infinite the chemical potential for water would be unaffected by the lipid and $\mu$ would have the value for bulk water. Also obvious is that $\mu$ must have this same value at $40 \%$ by weight since this is the proven equilibrium between the bulk lamella phase and pure water. The quantity $\Delta \mu$ indicated in the figure is the result of some sort of attractive force between layers since for $\mu$ greater than the value for bulk water but to the left of the maximum the system would prefer to loose water, allowing the layers to approach each other. In fact, it is well known that if one adds an ionic or charged lipid to an electrically neutral lipid like DPPC the effect is to add a repulsive component to the force between bilayers. The equilibrium water content increases above $40 \%$. For pure DPPC at high water content (i.e., $30-40 \%$ ) there is some attractive force between bilayers and we must ask how large.

Using different techniques to fix the chemical potential Elworthy [31] and Rand et al. [32] measured the water concentration as a function of chemical potential. They found that at approximately $30 \%$ weight water, which is well below the $40 \%$ value for equilibrium between bulk and free water, the attractive force per unit area between bilayers was of the order of 10 atmospheres. Although this may appear large at first glance, it is in fact very small when one considers the pressures required to modify the bulk properties of matter. For example, 10 atmospheres will reduce the melting temperature of ice less than $0.1^{\circ} \mathrm{C}$ and change the volume of paraffine by approximately $0.1 \%$. For all practical purposes, at temperatures above the gel transition, the interaction between bilayers in multilamellar samples of DPPC containing more than $30 \%$ weight water is negligible when measured against the type of forces that are required to influence molecular properties.

The same conclusion can be drawn from the concentration dependence of the gel transition temperature (see Fig. 5). Above about $20 \%$ water the temperature is independent of water content. Similarly, the latent heat associated with the gel transition in DPPC rises monotonically with increasing water content below about $25 \%$ water and then saturates at about $16 \mathrm{kT} / \mathrm{molecule}$ of lipid for higher water content. This is roughly three orders of magnitude larger than the difference between the chemical potential for water in the lipid at $25 \%$ weight and the value for bulk water. Again, this supports the idea that for these water concentrations the system is roughly equivalent to the ideal system of independent layers.

Jean-Pierre Le Pesant [33] carried out a Brillouin scattering study of the ultrasonic sound speeds in DPPC-water samples as a function of both water and temperature. If there is significant interaction between bilayers the smectic samples should have anisotropic elastic properties that would result in anisotropies in the ultrasonic velocities. Although measurable effects were found below the gel transition none were observable above the gel. Furthermore, the single, isotropic elastic constant that was measurable above the gel was independent of water above concentrations of the order of $13 \%$. Since the gel transition temperature and transition enthalpy depended on concentration up to $20-25 \%$ one is tempted to suggest that the elastic measurements 
are simply less sensitive. This may be true however, properties of the gel transition depend on both the high and low temperature phases and we do not have good measurements of the physical effects of water within the low temperature phase. In fact we know that below the gel transition the lipid-water is in equilibrium with bulk water at $25 \%$ by weight in contrast to $40 \%$ above the gel [34]. Thus the high and low temperature phases have some very different features and it is still possible that in the high temperature phases the interlayer interactions are negligible at $13 \%$ water while for the low temperature phase they are nonnegligible at all water contents.

Another experiment related to the interlayer water was developed in our laboratory by Winston Chan [35]. Brochard and de Gennes [36] demonstrated theoretically that a sinusoidal variation in the concentration of water, parallel to the bilayers, will decay exponentially with a time constant $\tau=\left(D q^{2}\right)^{-1}$, where $D$ is a phenomenological diffusion constant and $2 \pi / q$ is the spatial period or wavelength of the sinusoidal variation. Chan induced such a sinusoidal variation using the heat absorbed from two laser beams forming an interference pattern at the sample. By varying the angle between the two beams it was possible to vary $q$ and prove $\tau q^{2}$ was a constant, from which $D$ was determined. A theoretical model was developed relating $D$ to a phenomenological parameter that was identified as the effective thickness of free water. According to Chan this parameter is approximately zero at about $10 \%$ water and grows, nearly linearly, with increased water concentration becoming about $10 \AA$ somewhere between $25 \%$ and $35 \%$ water. Although it is not clear that the water concentration sufficient to make the Brillouin result above $T_{\mathrm{g}}$ independent of water, which corresponds Chan's parameter being between 1 and $3 \AA$, is sufficient that the layers can be regarded as non-interacting it is reasonable that $25 \%$ water, corresponding to more than $10 \AA$ of free water, should be sufficient.

5. Summary. - The principle theme that we have tried to develop is that amphiphillic monolayers are potentially useful for study of phase transitions, and the long-range order within two-dimensional systems. The most serious problem is the intrinsic metastability, or possible instability, when monolayers are subjected to high surface pressures. This is an essential difficulty with all two-dimensional systems and although it is not necessarily inevitable it must be considered in all experimental systems. The specific experiments discussed in relation to this point all employed phospholipids, however, similar phenomena is expected for nearly all amphiphillics and others, rather than lipids, may be better choices from this point of view.

A second theme, is that it is possible to form macroscopically oriented, multilamellar or smectic phases from amphiphillic-water mixtures (or for that matter from mixtures of amphiphillics and hydrophobic solvents) that present a number of interesting possible research directions. First, in line with the monolayer or two-dimensional phases, one should determine whether or not the smectics can be regarded as simple stacks of non-interacting two-dimensional systems. In the case of amphiphillic-water mixtures this may be true for the amphiphillic bilayers at high water content and some of the evidence supporting this was reviewed for DPPC. However, one should not overlook the possibility that even if DPPC is not suitable from this point of view, the bilayers of charged amphiphilles are more strongly repellent and will adsorb more interlayer water than neutral DPPC. Thus one might hope the bilayers in smectics of charged lipids will interact less.

Even if one can demonstrate that the bilayers are non-interacting above the gel transition it is far from clear that they will also be non-interacting below the transition. However, $\mathrm{X}$-ray experiments on lipids below $T_{\mathrm{g}}$ do reveal Bragg like spots that suggest two-dimensional order within the bilayers [34]. If the bilayers are non-interacting the smectic systems would be interesting models for two-dimensional phase transitions. On the other hand, if there are interlayer interactions below the gel the systems are probably in one or another smectic B phase and these have their own special interest. In particular the transition from a system of non-interacting twodimensional fluid bilayers to the three-dimensional smectic B could be studied as a function of water content.

Some of these ideas are rather naive and one should not try to push them too far without thinking about the other factors that determine the molecular organization. For example, simple soaps are charged amphiphillics and as one can see from the phase diagram in figure 3 addition of water to the lamella phase leads to an instability that results in the cylindrical phase. Earlier we suggested that this is related to the rather small polar group with a single hydrocarbon chain. Thus, one should not ignore intramolecular structure in deciding on the best system to study.

Another interesting possibility is that the polar parts of an amphiphillic will exhibit two-dimensional order and that these layers will be decoupled from adjacent polar layers by the hydrocarbon layers. We did not discuss this explicitly but Pomerantz et al. did present evidence for two-dimensional magnetic order in thin samples of $\mathrm{Mn}$ stearate [37].

Finally, the third and last theme is that multilamellar lipid bilayer samples incorporating ingredients found in biological membranes suggest numerous possibilities for spectroscopic studies of direct relevance to the biological function of these ingredients. In addition one might hope to develop techniques for forming aligned monodomain samples containing sufficient concentrations of these ingre- 
dients that X-ray or neutron diffraction studies will yield structural information.

Acknowledgments. - I would like to express my appreciation to the Scientific Organizing Committee of the 7th International Conference on Liquid Crystals for the invitation to present the lecture that this article is based on. I am also indebted to the students and scientific associates who, working in our laboratory, provided the results that were quoted. The financial support for that work was provided in part by the Joint Services Electronics Program (U.S. Army, Navy and Air Force) under Contract Number N00014-75-C-0648 and by the National Science Foundation under Grants numbered DMR76-01111 and DRM-76-22452.

\section{References}

[1] Adamson, A. W., Physical Chemistry of Surfaces (Wiley, N.Y.) 1976, 3rd Edition.

[2] TANFord, C., «The Hydrophobic Effect and the Organization of Living Matter ", Science 200 (1978) 1012.

[3] TANFord, C., The Hydrophobic Effect, Formation of Micelles and Biological Membranes (Wiley, New York) 1973.

[4] GiBbs, J. W., Trans. Conn. Acad. 3 (1876) 108, ibid 3 (1878) 343.

[5] Skoulius, A., Advan. Celloid and Interface Science 1 (1967) 79.

[6] Singer, S. J., Nicolson, G. L., Science 175 (1972) 720.

[7] Hinkle, P. C., McCarty, R. E., Sci. Am. 238 (1978) 104.

[8] Nagle, J. F., Scott, H. L., Phys. Today (1978) 38.

[9] Chapman, D., Quart. Rev. Biophys. 8 (1975) 2.

[10] Melchior, D. L., STeim, J. M., Ann. Rev. of Biophys. and Bioeng. 5 (1976) 205.

[11] Dervichian, D. G., Joly, M., J. Physique Radium 10 (1939) 375 ;

Dervichian, D., J. Chem. Phys. 7 (1939) 931.

[12] Albrecht, O., Gruler, H., Sackmann, E., J. Physique 39 (1978) 301.

[13] Landau, L. D. and Lifchitz, E. M., Statistical Physics (Pergamon, London) 1959, Chapt. 13.

[14] Mermin, N. D., Phys. Rev. 176 (1968) 250.

[15] See DE Gennes, P. G., The Physics of Liquid Crystals (Clarendon, Oxford) 1975 , p. 302 .

[16] Als-Nielsen, J., Birgeneau, R. J., Kaplan, M., Lifster, J. D., Safinya, C. F., Phys. Rev. Lett. 39 (1977) 1668.

[17] Berker, A. N., Ostlund, S., Putnam, F. A., Phys. Rev. B 17 (1978) 3650 .

[18] Halperin, B. I., Nelson, D., Phys. Rev. Lett. 41 (1978) 121.

[19] Pershan, P. S., Prost, J., J. Appl. Phys. 46 (1975) 2343.

[20] Gershfeld, N. L., TAuma, K., J. Colloid. and Interface Science 59 (1977) 597.
[21] MarceljA, S., Biophys. Biochem. Acta 367 (1976) 165.

[22] Bothorel, P., Belle, J., C. R. Hebd. Séan. Acad. Sci. 282 (1976) 437 ;

Bothorel, P., Belle, J., Lemaire, B., Chem. Phys. Lipuds 12 (1974) 96.

[23] Seelig, J., Niederberger, W., Biochemistry 13 (1974) 1585 ;

SeElig, A. and SeElig, J., Biochemistry 13 (1974) 4839.

[24] Mely, B., Charvolin, J., Keller, P., Chem. Phys. Lipids 15 (1975) 161.

[25] Powers, L., Clark, N. A., Proc. Nat. Acad. Sci. (USA) 72 (1972) 840.

[26] Powers, L., Pershan, P. S., Biophys. J. 20 (1977) 137.

[27] Chan, W., Pershan, P. S., Phys. Rev. Lett. 39 (1977) 1368 ; Biophys. $J$. (to be published).

[28] Rosenblatt, C. S., Pindak, R., Clark, N. A., Meyer, R. B., J. Physique 38 (1977) 1105.

[29] Sanford, Asher, A., Pershan, P. S. (submitted to J. Physique) 1978

[30] See Ref. [15], page 296.

[31] Elworthy, P. H., J. Chem. Soc. (1961) 5385 ; (1962) 4897.

[32] Le Neveu, D. M., Rand, R. P., Parsegian, V. A., Gingell, D., Biophys. J. 18 (1977) 209.

[33] Le Pesant, J.-P., Powers, L., Pershan, P. S., Proc. Nat. Acad. Sci. (USA) 75 (1978) 1792.

[34] Janiak, M. J., Small, D. M., Shipley, G. G., Biochemistry 15 (1976) 4575

[35] Chan, W., Pershan, P. S., Phys. Rev. Lett. 39 (1978) 1368 ; Biophysical $J$. (to be published).

[36] Brochard, F., De Gennes, P. G., Pramana, Suppl. No. 1 (1975) 1

[37] Pomerantz, M., Dacol, F. H., Segmüller, A., Phys. Rev. Lett. 40 (1978) 246. 